\title{
Co-infections with Chikungunya and Dengue Viruses, Guatemala, 2015
}

\section{Thomas Edwards, Leticia del Carmen Castillo Signor, Christopher Williams, Evelin Donis, Luis E. Cuevas, ${ }^{1}$ Emily R. Adams ${ }^{1}$}

We screened serum samples referred to the national reference laboratory in Guatemala that were positive for chikungunya or dengue viruses in June 2015. Co-infection with both viruses was detected by reverse transcription PCR in $46(32 \%)$ of 144 samples. Specimens should be tested for both arboviruses to detect co-infections.

C hikungunya virus (CHIKV) and dengue virus (DENV) are arboviruses currently circulating in Southeast Asia, Central and West Africa, the Pacific islands, and the Americas, and their transmission can occur simultaneously (1). DENV and CHIKV co-infections have been reported from 13 of 98 countries/territories to which the viruses are endemic and are more likely to occur in areas with high transmission intensity. Co-infection rates reported have ranged from $2 \%$ in Gabon to $34 \%$ in Nigeria (1-3).

The Americas are currently experiencing an unprecedented number of DENV infections that coincided with emergence of CHIKV infections. A total of 2.3 million DENV infections and 635,000 CHIKV infections were reported in this region in $2015(4,5)$. However, details of the frequency of co-infection are lacking (1), although a recent study involving 173 samples from Nicaragua that were positive for either virus found a co-infection rate of $22 \%(6)$.

Co-infections might be frequently missed by surveillance systems because Pan American Health Organization (PAHO) diagnostic algorithms indicate that DENV-positive samples do not need to be tested for CHIKV or other viruses and vice versa (7). The clinical role of co-infection is somewhat disputed because some (8) but not all $(1,9)$ studies reported an association between co-infection and disease severity for symptoms such as diarrhea (9) or hemorrhage (10).

Although Guatemala has had constant dengue transmission for $\geq 20$ years (11), the first cases of CHIKV infection were reported in September 2014. We report the

Author affiliations: Liverpool School of Tropical Medicine,

Liverpool, UK (T. Edwards, C. Williams, L.E. Cuevas, E.R.

Adams); Laboratorio Nacional de Salud Guatemala, Villa Nueva,

Guatemala (L. del Carmen Castillo Signor, E. Donis)

DOI: http://dx.doi.org/10.3201/eid2211.161017 proportion of patients co-infected with DENV and CHIKV and the association of co-infection with disease severity among patients referred for diagnosis to the National Health Laboratory (NHL) in Guatemala City, Guatemala, in June 2015. Because Zika virus was not introduced until November 2015, we did not test for this virus in this study.

\section{The Study}

Serum samples from febrile persons suspected of having arboviral infections are referred to the NHL in Guatemala for surveillance and confirmation purposes. For logistical reasons, most samples are received from 12 of the 22 districts in Guatemala, and most samples originate from Quetzaltenango, Guatemala, and Escuintla Districts, the largest urban centers in Guatemala.

We selected for screening a convenience set of consecutive samples received in June 2015, which is the peak transmission season for DENV, and reported as having positive results by reverse transcription PCR (RT-PCR) for either virus. Clinical data for patients were obtained from surveillance databases to compare patients with monoinfections or coinfections with DENV or CHIKV. All samples were obtained from patients $\leq 5$ days of symptom onset. Samples were obtained after patients provided consent and donated to laboratories for further testing. All samples were anonymous. Results of the study were not used for clinical management or surveillance purposes.

During June 2015, a total of 523 samples were tested for CHIKV at the NHL; $328(63 \%)$ were positive for CHIKV RNA by RT-PCR. A total of 514 samples were also tested by RT-PCR for DENV; 75 (9\%) were positive (Figure). Seventy-four of samples reported as positive for DENV RNA and 70 samples reported as positive for CHIKV RNA were available for further screening for the other virus.

Samples selected were consecutive positive samples from the beginning of the month; there were no additional exclusion or inclusion criteria. We included as many samples as possible within the time available. RNA was extracted by using a Viral RNA Mini Kit (QIAGEN, Manchester, UK) and tested by using the US Centers for Disease Control (CDC; (Atlanta, GA, USA) multiplex DENV RT-PCR and the CDC CHIKV 6856F/6981c/6919-FAM RT-PCR (12).

Twenty-five $(33.8 \%)$ of 74 DENV-positive samples were positive for CHIKV, and 21 (30\%) of $70 \mathrm{CHIKV-}$

${ }^{1}$ These senior authors contributed equally to this article. 

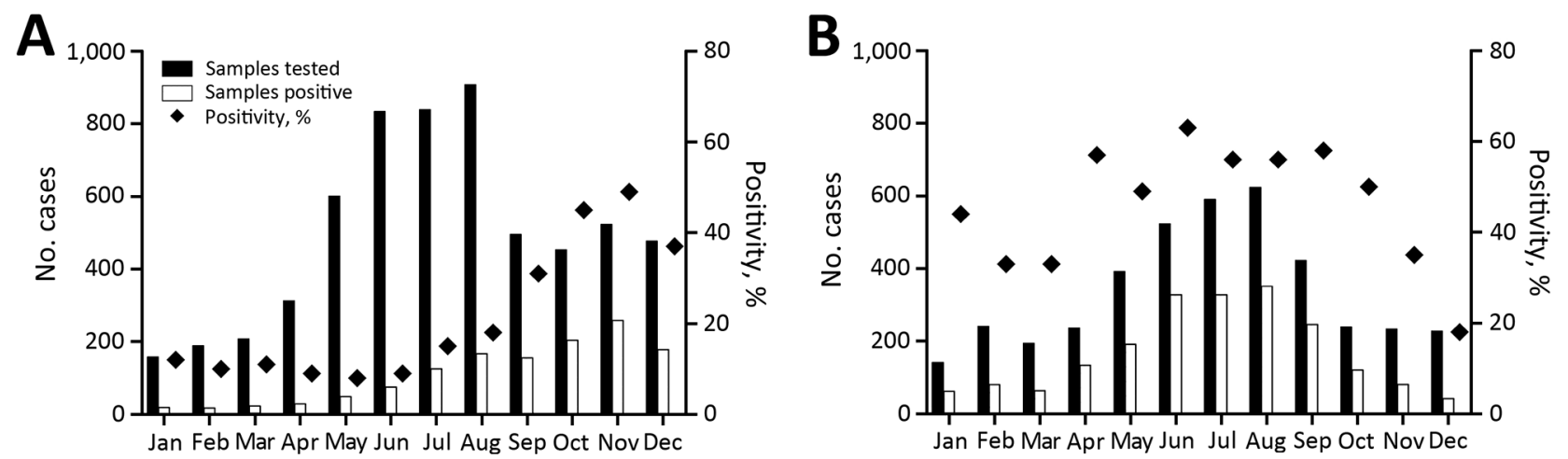

Figure. Positivity of samples tested for A) dengue virus and B) chikungunya virus, Guatemala, 2015.

positivesampleswerepositive forDENVRNA.Co-infection with CHIKV was detected in 4/9 samples containing DENV-1, 40/76 samples containing DENV-2, 0/3 samples containing DENV-3 and 2/7 samples containing DENV-4.

Clinical characteristics of patients with CHIKV and DENV monoinfections and co-infections were similar (Table). Patients co-infected with CHIKV and DENV were more likely to have a rash than those with DENV monoinfections $(p=0.02)$ and were more likely to be hospitalized than those with CHIKV monoinfections $(p=0.002)$. All other associations were not statistically significant.

\section{Conclusions}

Our findings must be viewed with caution because of the limitations of this study. The study was not powered to analyze clinical associations with a high robustness because of the small number $(n=46)$ of co-infected samples tested. Also, because samples were selected at a reference laboratory and only symptomatic cases were tested, the prevalence of co-infection might be different in a less selected population.

The PAHO algorithm recommends screening clinical specimens by using consecutive assays and ending screening once a pathogen is identified. CDC in turn recommends simultaneously conducting RT-PCRs for DENV, CHIKV, and Zika virus. Emergence of Zika virus in Latin America has further complicated diagnosis of arboviral infections, and simultaneous co-infections with all 3 arboviruses have been reported (13). Although the PAHO approach misses co-infections, the CDC approach is costlier.

Guatemala used the PAHO algorithm because of a shortage of consumable supplies. On further testing, 25 $(33.8 \%)$ of 74 DENV-positive samples were positive for CHIKV, and 21 (30\%) of $70 \mathrm{CHIKV-positive} \mathrm{samples} \mathrm{were}$ positive for DENV. Because Guatemala reported 49,043 cases of DENV and CHIKV infections in 2015, it is likely that the algorithm missed a large number of co-infections.

\begin{tabular}{|c|c|c|c|c|c|}
\hline \multirow[b]{2}{*}{ Characteristic } & \multirow{2}{*}{$\begin{array}{c}\text { CHIKV } \\
\text { moninfection }\end{array}$} & \multirow{2}{*}{$\begin{array}{c}\text { DENV } \\
\text { monoinfection }\end{array}$} & \multirow{2}{*}{$\begin{array}{c}\text { DENV/CHIKV } \\
\text { co-infection }\end{array}$} & \multicolumn{2}{|c|}{$p$ value } \\
\hline & & & & CHIKV vs co-infection & DENV vs co-infection \\
\hline No. samples tested & 49 & 49 & 46 & & \\
\hline \multicolumn{6}{|l|}{ Sex } \\
\hline$M$ & $15 / 48(31)$ & $23 / 45(51)$ & $21 / 46(46)$ & 0.2 & 0.6 \\
\hline $\mathrm{F}$ & $33 / 48$ (69) & $22 / 45$ (49) & $25 / 46(54)$ & 0.2 & 0.6 \\
\hline \multicolumn{6}{|l|}{ Age, $y$} \\
\hline$\leq 1$ & $2 / 48(4.2)$ & $3 / 45(7)$ & $5 / 46(11)$ & 0.2 & 0.7 \\
\hline$\overline{2}-16$ & $15 / 48(31)$ & $26 / 45(58)$ & $19 / 46(41)$ & 0.4 & 0.1 \\
\hline $17-30$ & $13 / 48(27)$ & $6 / 45(13)$ & $9 / 46(20)$ & 0.4 & 0.5 \\
\hline $31-60$ & $14 / 48(29)$ & $9 / 45(20)$ & $10 / 46(22)$ & 0.5 & 1 \\
\hline$\geq 60$ & $4 / 48(8.3)$ & $1 / 45(2)$ & $3 / 46(7)$ & 1 & 0.6 \\
\hline \multicolumn{6}{|l|}{ Sign/symptom } \\
\hline Hemorrhage & $7 / 49(14)$ & $11 / 44(25)$ & $8 / 44(18)$ & 0.8 & 0.6 \\
\hline Arthralgia/myalgia & $41 / 44(93)$ & 36/42 (86) & $35 / 45(77)$ & 0.07 & 0.4 \\
\hline Fever & $43 / 48(90)$ & $41 / 42$ (98) & $46 / 46(100)$ & 0.06 & 0.5 \\
\hline Rash & $31 / 48(65)$ & $11 / 43(26)$ & $23 / 44(52)$ & 0.3 & 0.02 \\
\hline Nausea & $26 / 46(57)$ & $31 / 44(70)$ & $22 / 44(50)$ & 0.7 & 0.08 \\
\hline Vomiting & $13 / 46(28)$ & $21 / 43(49)$ & $16 / 45(36)$ & 0.5 & 0.3 \\
\hline Diarrhea & $3 / 18(17)$ & 10/34 (29) & $6 / 25(24)$ & 0.7 & 0.8 \\
\hline Headache & $40 / 47(85)$ & $35 / 42(83)$ & $40 / 44(91)$ & 0.5 & 0.3 \\
\hline Hospitalization & $0 / 48(0)$ & $16 / 45(36)$ & $8 / 46$ (17) & 0.002 & 0.09 \\
\hline Death & $0 / 48(0)$ & $4 / 45(9)$ & $2 / 46(4)$ & 0.2 & 0.4 \\
\hline
\end{tabular}

"Values are no. positive/no. tested (\%) unless otherwise indicated. CHIKV, chikungunya virus; DENV, dengue virus. 
Patients with single and dual infections had similar clinical manifestations in this limited study.

The frequency of arbovirus coinfections in Guatemala is high. Simultaneous screening for DENV, CHIKV, and Zika virus in disease-endemic areas would improve the quality of arboviral surveillance and potentially aid in clinical management of the disease.

This study was supported by a Medical Research Council Confidence in Concept award (MC-PC_14111).

Dr. Edwards is a postdoctoral research associate at the Research Centre for Drugs and Diagnostics in the Liverpool School of Tropical Medicine, Liverpool, UK. His primary research interests are design and implementation of molecular tools for diagnosing arboviral infections and antimicrobial drug resistance.

\section{References}

1. Furuya-Kanamori L, Liang S, Milinovich G, Soares Magalhaes RJ, Clements AC, Hu W, et al. Co-distribution and co-infection of chikungunya and dengue viruses. BMC Infect Dis. 2016;16:1-11.

2. Caron M, Paupy C, Grard G, Becquart P, Mombo I, Nso BB, et al. Recent introduction and rapid dissemination of chikungunya virus and dengue virus serotype 2 associated with human and mosquito coinfections in Gabon, central Africa. Clin Infect Dis. 2012;55:e45-53. http://dx.doi.org/10.1093/cid/cis530

3. Baba M, Logue CH, Oderinde B, Abdulmaleek H, Williams J, Lewis J, et al. Evidence of arbovirus co-infection in suspected febrile malaria and typhoid patients in Nigeria. J Infect Dev Ctries. 2013;7:51-9. http://dx.doi.org/10.3855/jidc. 2411

4. Pan American Health Organization. Number of reported cases of dengue and severe dengue (SD) in the Americas, by country, January 15, 2016 (EW 52) [cited 2016 Apr 23]. http://www.paho. org/hq/index.php?option=com_topics\&view $=$ rdmore $\&$ cid $=6290 \& \mathrm{I}$ temid $=40734 \&$ lang $=$ en

5. Pan American Health Organization. Number of reported cases of chikungunya fever in the Americas, December 31, 2015 (EW 52) [cited 2014 Apr 23]. http://www.paho.org/hq/index. php?option $=$ com_topics\&view $=$ readall\&cid $=5927 \&$ Itemid $=40931$ \&lang=en

6. Waggoner JJ, Gresh L, Mohamed-Hadley A, Ballesteros G, Davila MJV, Tellez Y, et al. Single-reaction multiplex reverse transcription PCR for detection of Zika, chikungunya and dengue viruses. Emerg Infect Dis. 2016;22:1295-7. http://dx.doi.org/10.3201/eid2207.160326

7. Pan American Health Organization. Zika virus (ZIKV) surveillance in the Americas: recommendations for laboratory detection and diagnosis, 2016 [cited 2016 May 1]. http://www.paho.org/hq/index. php?option $=$ com_docman\&task $=$ doc_details\&gid $=30176 \&$ Itemid $=$ 270\&lang $=$ en

8. Gandhi SB, Kulkarni K, Godbole M, Dole SS, Kapur S, Satpathy P, et al. Dengue and chikungunya co-infection associated with more severe clinical disease than mono-infection. International Journal of Healthcare and Biomedical Research. 2015;3:117-23 [cited 2016 Aug 25]. http://ijhbr.com/pdf/April\%20 2015\%20117-123.pdf

9. Taraphdar D, Sarkar A, Mukhopadhyay BB, Chatterjee S. A comparative study of clinical features between monotypic and dual infection cases with chikungunya virus and dengue virus in West Bengal, India. Am J Trop Med Hyg. 2012;86:720-3. http://dx.doi.org/10.4269/ajtmh.2012.11-0704

10. Chahar HS, Bharaj P, Dar L, Guleria R, Kabra SK, Broor S. Co-infections with chikungunya virus and dengue virus in Delhi, India. Emerg Infect Dis. 2009;15:1077-80. http://dx.doi.org/10.3201/eid1507.080638

11. Wilson ME, Chen LH. Dengue in the Americas. Dengue Bull. 2002;26:44-61.

12. Lanciotti RS, Kosoy OL, Laven JJ, Panella AJ, Velez JO, Lambert AJ, et al. Chikungunya virus in US travelers returning from India, 2006. Emerg Infect Dis. 2007;13:764-7. http://dx.doi.org/10.3201/eid1305.070015

13. Villamil-Gómez WE, González-Camargo O, Rodriguez-Ayubi J, Zapata-Serpa D, Rodriguez-Morales AJ. Dengue, chikungunya and Zika co-infection in a patient from Colombia. J Infect Public Health. 2016;9:684-6. http://dx.doi.org/10.1016/j. jiph.2015.12.002

Address for correspondence: Thomas Edwards, Research Centre for Drugs and Diagnostics, Liverpool School of Tropical Medicine, Pembroke Pl, Liverpool L3 5QA, UK; email: thomas.edwards@1stmed.ac.uk

\title{
PubMed Centra
}

\section{PublMed}

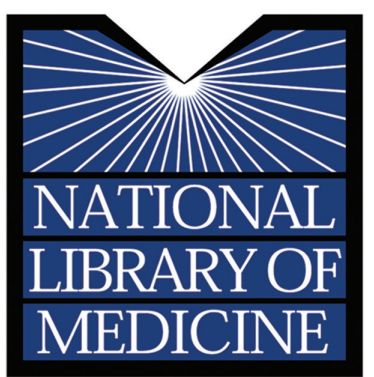

\section{Find Emerging Infectious Diseases content in the digital archives of the National Library of Medicine}

\author{
www.pubmedcentral.nih.gov
}

